\title{
Uniform approximation with Diophantine side-conditions of continuous functions
}

\author{
By Matts Håstad
}

This article is composed of two entirely different parts. The first part deals with approximation of real continuous functions in one variable by rational functions whose zeros and poles all belong to certain prescribed sets. The second part is about approximation of real continuous functions in several variables by polynomials with integral coefficients. Two general theorems are proved and finally some special results are discussed. Fekete has completely analysed the problem in one variable [3].

\section{I}

Theorem 1: Each positive continuous function $f(x)$ admits uniform approximation on $I=[-1,+1]$ by the quotient of two real polynomials, whose zeros belong to two given sets $P$ and $\bar{P}$ satisfying:

1) $\bar{P}$ is the image of $P$ under reflection in the real axis.

2) $P($ and $\bar{P})$ is situated on a line segment whose extension does not intersect $I$ perpendicularly.

3) $P($ and $\bar{P})$ has a subset $P^{\prime}\left(\right.$ and $\left.\bar{P}^{\prime}\right)$ which is dense in itself (i.e. $P^{\prime} \subset$ the set of all the accumulation points of $\left.P^{\prime}\right)$.

Proof: We use the following well-known theorem: The set $M$ is dense in $C(-1,1)$ if and only if each linear functional in $C(-1,1)$ which is zero for every element of $M$ is identically zero. A real functional $L(f)$ in $C(-1,1)$ may be written as

$$
L(f)=\int_{-1}^{+1} f(x) d \mu(x)
$$

where $\mu$ is a real function of bounded variation. We define $M$ as the set of functions $\left\{e^{i \varphi} /(x-a)+e^{-i \varphi} /(x-\bar{a})\right\}$ for all $a=\alpha+i \beta \in P^{\prime} ; \varphi$ is the argument of the line $l$ to which $a$ belongs. The assumption $L(f)=0$ for $f \in M$ then implies that the function

$$
u(\alpha, \beta)=\int_{-1}^{+1}\left(\frac{e^{i \varphi}}{x-a}+\frac{e^{-i \varphi}}{x-\bar{a}}\right) d \mu(x)
$$

vanishes for all $a \in P^{\prime}$. But $u(\alpha, \beta)$ being the real part of an analytic function $\psi(a)$, holomorphic for $a \notin I$, is harmonic, and we consequently have $u(\alpha, \beta)=0$ on the whole line $l$ except in its possible intersection with $I$. Using the principle of reflection we get: 


\section{HÅSTAD, Uniform approximation of continuous functions}

1) The line $l$ does not intersect $I$. Then $\psi(a)$ is holomorphic in one of the halfplanes that $l$ forms. According to the principle of reflection, $\psi(a)$ must be holomorphic in the whole plane which gives $d \mu \equiv 0$.

2) lintersects $I, \varphi \neq \pm \frac{\pi}{2} . \psi(a)$ is then holomorphic in either of two points symmetrically situated to $l$. From the principle of reflection we conclude that $\psi(a)$ is holomorphic in the whole plane, except possibly in $l$ :s intersection $\left(x_{0}, 0\right)$ with $I$. Consequently $d \mu$ reduces to a point mass in this point. We have $\psi(a)=k e^{i \varphi} /$ $\left(x_{0}-a\right)$, where $k$ is real. If $a \in l$ we have $x_{0}-a=\varrho e^{i \varphi}$ ( $\varrho$ real) and accordingly $\operatorname{Re}(\psi(a))=k / \varrho$. But $\operatorname{Re}(\psi(a))=0$ if $a \in l$ which gives $k=0$ and hence $d \mu \equiv 0$.

3) $l$ intersects $I$ perpendicularly. This is the case excluded in theorem 1. (See below.)

We consequently know that each continuous function $g(x)$ may be approximated uniformly by an expression

$$
g_{1}(x)=\sum_{1}^{m} A_{k}\left(\frac{e^{i \varphi}}{x-a_{k}}+\frac{e^{-i \varphi}}{x-\bar{a}_{k}}\right)
$$

where $A_{k}$ are real and $a_{k} \in P^{\prime}$. We choose $g(x)=\log f(x)$ where $f(x)$ is the positive continuous function in theorem 1. Given $\varepsilon>0$, we take $a_{k}^{\prime}=a_{k}+\delta_{k} e^{i \varphi}\left(\delta_{k}\right.$ real) where $a_{k}^{\prime} \in P^{\prime}$ and

$$
\left|\delta_{k}\right| \leq \min \left(\frac{\varepsilon}{4 G m}, \frac{\varepsilon}{4 G^{2}\left(\left|A_{k}\right|+1\right) m}, \frac{1}{2 G}\right) .
$$

Here $G$ is the maximum of $1 /|x-a|$ for $a_{k} \in P^{\prime}$ and $x \in I$, and the choice is evidently possible because $P^{\prime}$ is dense in itself. Consider one of the $2 m$ terms in $g_{1}(x)$, say $A_{k} e^{i p} /\left(x-a_{k}\right)$. Putting $n_{k}=\left[A_{k} / \delta_{k}\right]$ we get by the aid of the logarithmic expansion

$$
\begin{aligned}
\left|\frac{A_{k} e^{i \varphi}}{x-a_{k}}-n_{k} \log \frac{x-a_{k}}{x-a_{k}^{\prime}}\right| & \leq \frac{1}{\left|x-a_{k}\right|}\left|A_{k}-n_{k} \delta_{k}\right|+\left|\frac{n_{k} \delta_{k} e^{i \varphi}}{x-a_{k}}-n_{k} \log \frac{x-a_{k}}{x-a_{k}^{\prime}}\right| \leq \\
& \leq G\left|\delta_{k}\right|+\frac{\left|n_{k}\right| G^{2} \delta_{k}^{2}}{2\left(1-\left|\delta_{k}\right| G\right)} \leq \frac{\varepsilon}{2 m} .
\end{aligned}
$$

Consequently $g_{1}(x)$ may be approximated with an error $<\varepsilon$ by the sum

$$
\sum_{1}^{m} n_{k}\left(\log \frac{x-a_{k}}{x-a_{k}^{\prime}}+\log \frac{x-\bar{a}_{k}}{x-\bar{a}_{k}^{\prime}}\right)
$$

which gives an approximation of $f(x)$ by the rational function

$$
\prod_{1}^{m}\left[\frac{\left(x-a_{k}\right)\left(x-\bar{a}_{k}\right)}{\left(x-\bar{a}_{k}^{\prime}\right)\left(x-\bar{a}_{k}^{\prime}\right)}\right]^{n_{k}} \text {. }
$$

This function has real coefficients and its zeros and poles all belong to $P$ and $\bar{P}$. Hence theorem 1 is proved.

By extending $P$ and $\bar{P}$ we can get rid of the condition $f(x)>0$. We see at once that if a continuous function, which is both positive and negative in $I$, shall admit approximation by a rational function, this must also have zeros in the interval. We have the following theorem: 
Theorem 2: Each continuous function admits uniform approximation in I by the quotient of two polynomials, the zeros of the numerator belonging to $I$ and to the given sets $P$ and $\bar{P}$ in theorem 1 and those of the denumerator to $P$ and $\bar{P}$.

Proof: Take an $\varepsilon>0$. According to Weierstrass $f(x)$ may be approximated by a polynomial $g(x)$ with an error $<\frac{\varepsilon}{3}$. Suppose

$$
g(x)=\left(x-x_{1}\right) \ldots\left(x-x_{n}\right) h(x)=k(x) \cdot h(x)
$$

where $x_{1}, x_{2} \ldots x_{n} \in I$ and $h(x)>0$ in $I$. Suppose $\max _{x \in I} k(x)=k_{1}$ and define

$$
l(x)= \begin{cases}h(x) & \text { if } h(x)>\frac{\varepsilon}{3 k_{1}} \\ \frac{\varepsilon}{3 k_{1}} & \text { if } h(x) \leqslant \frac{\varepsilon}{3 k_{1}}\end{cases}
$$

$l(x)$ is positive and continuous and consequently admits approximation by the rational function $m(x)$ in accordance with theorem 1. The error in the approximation can be made $<\frac{\varepsilon}{3 k_{1}}$. If we finally show that $m(x) \cdot k(x)$ approximates $f(x)$ with an error $<\varepsilon$, we have proved theorem 2. In fact we have:

$$
\begin{gathered}
|f(x)-m(x) k(x)| \leqslant|f(x)-g(x)|+|k(x)||h(x)-l(x)|+|k(x)||l(x)-m(x)| \leqslant \\
\leqslant\left\{\begin{array}{lc}
\frac{\varepsilon}{3}+0+k_{1} \frac{\varepsilon}{3 k_{1}}=\frac{2}{3} \varepsilon & \text { if } h(x)>\frac{\varepsilon}{3 k_{1}} \\
\frac{\varepsilon}{3}+k_{1} \frac{\varepsilon}{3 k_{1}}+k_{1} \frac{\varepsilon}{3 k_{1}}=\varepsilon & \text { if } h(x) \leqslant \frac{\varepsilon}{3 k_{1}} .
\end{array}\right.
\end{gathered}
$$

If all the zeros are situated on a line which intersects $I$ perpendicularly, the rational expression becomes symmetric with respect to this line and then only those continuous functions may be approximated that have this symmetry. $I=[-1,1]$ may further be replaced by any finite interval and the line segments may be replaced by one segment $(\neq I)$ on the real axis.

\section{II}

Theorem 3: $A$ necessary and sufficient condition that each continuous function $f(x, y \ldots)$ in several real variables admits uniform approximation in a compact set $A$ by a polynomial whose coefficients are rational integers, is the existence of a polynomial with integral coefficients, $u(x, y \ldots)$ such that

$$
\text { 1) }|u(x, y \ldots)|<1 \text { in } A ; \quad \text { 2) } u(x, y \ldots) \neq 0 \text { in } A \text {. }
$$

In the following we suppose, for the sake of simplicity, that the number of variables is two. The results and the proofs are the same for any number of variables. From here on we call a polynomial with integral coefficients an I-polymonial. The class of all compact sets satisfying 1 ) and 2) is called $M$. 


\section{HÅSTAD, Uniform approximation of continuous functions}

Proof: a) The condition is necessary:

In order that the function $f(x, y) \equiv \frac{1}{2}$ shall admit uniform approximation in $A$ by an I-polynomial $u(x, y)$, this must satisfy 1) and 2) above. Hence there exists a $u(x, y)$ with the required properties.

b) The condition is sufficient:

We use the following lemma, principally well-known, which is given here without proof [1].

Lemma 1: Suppose that the family $T$ of continuous functions $t(x, y)$ defined in $A$ has the property that for any two functions $t_{1}(x, y)$ and $t_{2}(x, y) \in T$, max $\left(t_{1}, t_{2}\right)$ and $\min \left(t_{1}, t_{2}\right)$ also belong to $T$. Then a function $f(x, y)$, continuous in $A$, admits uniform approximation by functions $t(x, y)$ if and only if for any two points $\left(x_{0}, y_{0}\right)$ and $\left(x_{1}, y_{1}\right)$ in $A$ and any $\varepsilon>0$, there is a function $t(x, y)$ such that

$$
\alpha)|f(x, y)-t(x, y)|<\varepsilon \text { for }\left(x_{0}, y_{0}\right) \text { and }\left(x_{1}, y_{1}\right)
$$

For the proof of theorem 3 above, take as the family $T$ in the lemma all continuous functions admitting approximation by polynomials without low powers $p\left(u_{1}, u_{2}, u_{3}\right)$ in the three variables $u_{1}=u(x, y), u_{2}=\left(x-n_{1}\right)(u(x, y))^{m_{1}}$ and $u_{3}=$ $=\left(y-n_{2}\right)(u(x, y))^{m_{2}}, n_{1}, n_{2}, m_{1}, m_{2}$ are integers, the lines $x=n_{1}$, and $y=n_{2}$ do not intersect $A$, and $u(x, y)$ is an I-polynomial satisfying the conditions 1) and 2) of the theorem. The integers $m_{1}$ and $m_{2}$ may be chosen so large that $\left|u_{2}\right|<1$ and $\left|u_{3}\right|<1$ in $A$. The coefficients of the polynomial $p$ shall be zero for the terms with lower degree than $n$ (fixed).

By writing $\max \left(t_{1}, t_{2}\right)=\frac{1}{2}\left(t_{1}+t_{2}+\left|t_{1}-t_{2}\right|\right)$ and $\min \left(t_{1}, t_{2}\right)=\frac{1}{2}\left(t_{1}+t_{2}-\left|t_{1}-t_{2}\right|\right)$ it will be enough to show that $t \in T \Rightarrow|t| \in T$. If $t$ is approximated by a polynomial $p$, then $|p|$ approximates $|t|$. According to Weierstrass we know however that $|p|$ may be approximated by a polynomial $q(p)$ in $p$, vanishing for $p=0$. Hence $q(p)$ will lack terms of low degrees and we have proved that min and max belong to $T$.

It is easily seen that the set of polynomials $\left(u_{1}, u_{2}, u_{3}\right)$ separates the points of $A$, i.e. given two different points $\left(x_{0}, y_{0}\right)$ and $\left(x_{1}, y_{1}\right)$ there is at least one polynomial $u_{i}$ such that $u_{i}\left(x_{0}, y_{0}\right) \neq u_{i}\left(x_{1}, y_{1}\right)$. Using the notations

$$
\begin{aligned}
& u_{i}=v ; v\left(x_{0}, y_{0}\right)=v_{0} ; v\left(x_{1}, y_{1}\right)=v_{1} \\
& f_{0}=f\left(x_{0}, y_{0}\right) / v_{0}^{n} ; f_{1}=f\left(x_{1}, y_{1}\right) / v_{1}^{n}
\end{aligned}
$$

we construct the polynomial without low powers

$$
p(v)=\left\{f_{0}+\frac{f_{1}-f_{0}}{v_{1}-v_{0}}\left(v-v_{0}\right)\right\} v^{n}
$$

which satisfies the condition $\alpha$ ) with $\varepsilon=0$. Thus this family has all the properties desired in the lemma so that each continuous function may be approximated by a polynomial $p\left(u_{1}, u_{2}, u_{3}\right)$ without low powers. We have further:

$$
\begin{aligned}
p\left(u_{1}, u_{2}, u_{3}\right)=a_{1} u_{1}^{n}+a_{2} u_{1}^{n-1} u_{2}+ & a_{3} u_{1}^{n-1} u_{3}+\cdots= \\
& =\left[a_{1}\right] u_{1}^{n}+\left[a_{2}\right] u_{1}^{n-1} u_{2}+\left[a_{3}\right] u_{1}^{n-1} u_{3}+\cdots+R_{n},
\end{aligned}
$$


where $R_{n}$ is a polynomial whose coefficients are absolutely less than 1. Because $\max _{k}\left|u_{k}\right|=s<1$ for $(x, y) \in A$ we get

$$
\left|R_{n}\right| \leqslant\left|u_{1}^{n}\right|+\left|u_{1}^{n-1} u_{2}\right|+\cdots=\sum\left|u_{1}^{b_{1}} u_{2}^{b_{2}} u_{3}^{b_{3}}\right|
$$

where the sum is extended over all combinations of non-negative integers whose sum is $\geqq n$. Hence [2]

$$
\left|R_{n}\right|<\sum_{k=n}^{\infty}\left(\begin{array}{c}
k+2 \\
2
\end{array}\right) s^{k}
$$

and this series being convergent for $|s|<1$ we can make $R_{n}$ arbitrarily small by choosing $n$ sufficiently large. Thus we have an approximating I-polynomial and theorem 3 is proved.

By making some restrictions concerning the continuous functions, which are to be approximated, the set where approximation is possible may be extended. Let $M^{\prime}$ denote the class of compact sets $B$ such that there exist an I-polynomial $u$ satisfying the conditions

$$
\text { 1') } \left.|u(x, y)|<1 \text { in } B \quad 2^{\prime}\right) u(x, y) \neq 0 .
$$

Each set $B \in M^{\prime}$ has a subset $N(B)$ such that $\left(x_{0}, y_{0}\right) \in N(B)$ implies $u\left(x_{0}, y_{0}\right)=0$ for every I-polynomial satisfying $\left.1^{\prime}\right)$ and $2^{\prime}$ ). To $N(B)$ belong in particular all points in $B$ with integral coordinates, for in such a point $u(x, y)$ must be an integer absolutely less than one. Hence $u(x, y)=0$.

Theorem 4: In $B \in M^{\prime}$ those and only those continuous functions admit uniform approximation by I-polynomials for which there exist an I-polynomial $p(x, y)$ such that $f(x, y)=p(x, y)$ for $(x, y) \in N(B)$.

For the proof we need the following lemma:

Lemma 2: There exists in $B$ an I-polynomial $u^{*}$ such that $\left|u^{*}(x, y)\right|<1$ in $B$ and $u^{*}(x, y) \neq 0$ in $B-N(B)$.

Proof of the lemma: The ideal generated by all I-polynomials satisfying 1') is called $F$. It is not the zero ideal and, being an ideal in a Noetherian ring, it is finitely generated by a set of polynomials $\left\{u_{i}\right\}, i=1,2 \ldots, n$, where we may assume $\left|u_{t}\right|<1$. Now consider the polynomial

$$
u^{*}=\sum_{1}^{n} u_{i}^{2 m_{i}}
$$

By choosing the integers $m_{i}$ sufficiently large we can attain $\left|u^{*}\right|<1$ in $B$ and evidently $u^{*} \neq 0$ in $B-N(B)$. For otherwise $u_{i}(x, y)=0, i=1,2, \ldots, n$, for some point $(x, y) \in B-N(B)$ which implies $u(x, y)=0$ for all $u \in F$, contradicting the definition of $N(\mathrm{~B})$.

Proof of the theorem 4: We first prove that the functions mentioned in the theorem admit approximation. It is evidently enough to show that $g(x, y)=f(x, y)-$ $-p(x, y)$ admits approximation. But having shown the existence of $u^{*}$ the proof of the sufficiency of theorem 3 will be valid also in this case. The only thing 


\section{HÅSTAD, Uniform approximation of continuous functions}

that has to be modified is the choice of the polynomial $p(v)$ if one of or both the points $\left(x_{0}, y_{0}\right)$ and $\left(x_{1}, y_{1}\right)$ belong to $N(B)$. In that case however we define $f_{i} \equiv 0 \quad(i=1,2)$ and $p \equiv 0$ respectively. Suppose then that $f(x, y)$ may be approximated uniformly by $I$-polynomials in $B$. Then there exist $p_{1}(x, y)$ and $p_{2}(x, y) \neq$ 丰 $p_{1}(x, y)$ such that $\left|f(x, y)-p_{i}(x, y)\right|<\frac{1}{2}, i=1,2$. Put $\bar{u}=p_{1}-p_{2}$. We have $|\bar{u}| \leqslant$ $\leqslant\left|p_{1}-f\right|+\left|f-p_{2}\right|<1$ which implicates $\bar{u}(\bar{x}, \bar{y})=0$ for $(\bar{x}, \bar{y}) \in N(B)$. That means that all $I$-polynomials that approximate $f(x, y)$ with an error $<\frac{1}{2}$ have the same values in $N(B)$. In order that $f(x, y)$ shall admit uniform approximation we must have $f(\bar{x}, \bar{y})=p(\bar{x}, \bar{y})$ if $(\bar{x}, \bar{y}) \in N(B)$. That completes the proof of theorem 4 .

We shall not give the general condition on $M$ and $M^{\prime}$ here, but only some special cases which can be brought back to approximation on the line. According to the work of Fekete [3], approximation by I-polynomials is possible in an interval if its length is $<4$, or more generally, in a set in the complex plane if its capacity is $<1$. The set $N$ is here composed of those algebraic integers whose conjugates all belong to the interval (set).

Theorem 5: $A$ rectangle $R^{\prime}: a \leqslant x \leqslant b, c \leqslant y \leqslant d$ parallel to the coordinate-axes and with sides $<4$ belongs to $M^{\prime}$, and $N\left(R^{\prime}\right)=N_{x}(a, b) \times N_{y}(c, d)$.

Proof: Take $u_{0}(x, y)=q^{2}(x)+r^{2}(y)$, where $q(x)<\frac{1}{\sqrt{2}}$ and $q(x) \neq 0$ when $x \notin$ $N_{x}(a, b)$ and correspondingly for $y$. The existence of $q(x)$ and $r(y)$ follows from lemma 2. Thus we have $\left|u_{0}(x, y)\right|<1$ in $R^{\prime}$. This proves the first assertion.

From the above we get $N\left(R^{\prime}\right) \subseteq N_{x}(a, b) \times N_{y}(c, d)$. In order to prove $N\left(R^{\prime}\right) \supseteq$ $N_{x}(a, b) \times N_{y}(c, d)$ suppose $\left(\bar{x}_{1}, \bar{y}_{1}\right) \in N_{x}(a, b) \times N_{y}(c, d)$. The number $\bar{x}_{1}$, which is an algebraic integer, has the conjugates $\bar{x}_{2}, \bar{x}_{3} \ldots, \bar{x}_{n}$ all of which belong to $N_{x}(a, b)$. The conjugates of $\bar{y}_{1}$ are $\bar{y}_{2}, \bar{y}_{3}, \ldots, \bar{y}_{m}\left(\in N_{y}(c, d)\right)$. Form

$$
U(z)=\prod_{\substack{i=1 \\ k=1}}^{n_{j} m}\left(z-u\left(\bar{x}_{i}, \bar{y}_{k}\right)\right)
$$

where $u(x, y)$ is any $I$-polynomial satisfying $|u|<1$ in $R^{\prime} . U(z)$ is a symmetric expression in $\bar{x}_{1}, \bar{x}_{2}, \ldots, \bar{x}_{n}$ and $\bar{y}_{1}, \bar{y}_{2}, \ldots, \bar{y}_{m}$, whose elementary symmetric functions are integers and consequently $U(z)$ is an $I$-polynomial in $z$. Hence $z^{(1)}=u\left(\bar{x}_{1}, \bar{y}_{1}\right)$, being a root of $U(z)=0$, is an algebraic integer and has the conjugates $z^{(2)}, z^{(3)}, \ldots, z^{(p)}$, which evidently are to be found among the $u\left(\bar{x}_{i}, \bar{y}_{k}\right),(i=1,2,3 \ldots n, k=1,2,3 \ldots m)$. This gives $Z=\prod_{\nu=1}^{p}\left|z^{(v)}\right|<1$ because each $z^{(v)}<1 . Z$ is an integer, hence $Z=0$. We must then have $u\left(\bar{x}_{1}, \bar{y}_{1}\right)=0$, but $\left(\bar{x}_{1}, \bar{y}_{1}\right)$ was any element of $N_{x}(a, b) \times N_{y}(c, d)$. This implicates $N_{x}(a, b) \times N_{y}(c, d) \subseteq N\left(R^{\prime}\right)$ and the proof is complete.

Theorem 6: Any rectangle $R^{\prime \prime}: a \leqslant x \leqslant b, c \leqslant y \leqslant d$, whose sides are parallel to the axes and such that $b-a<4, d-c=k \geqslant 4$ belongs to $M^{\prime}$. Then $(x, y) \in N\left(R^{\prime \prime}\right)$ if and only if $x \in N_{x}(a, b)$.

Proof: Put $u_{0}(x, y)=(y-n) q^{m}(x)$ where $n$ is an integer $>d$ and $|q(x)|<1$ in $(a, b)$ and $q(\bar{x})=0$ if and only if $\bar{x} \in N_{x}(a, b)$. By choosing $m$ sufficiently large we get $\left|u_{0}(x, y)\right|<1$ for any $(x, y) \in R^{\prime \prime}$. From the definition of $u_{0}(x, y)$ it follows that if $\hat{x} \notin N_{x}(a, b)$ we have $(\hat{x}, y) \notin N\left(R^{\prime \prime}\right)$ and it remains to be shown that $\bar{x} \in$ 
$N_{x}(a, b) \Rightarrow(\bar{x}, y) \in N\left(R^{\prime \prime}\right)$. Take any $I$-polynomial $u(x, y)$ such that $|u(x, y)|<1$ in $R^{\prime \prime}$ : $u(x, y)=q_{n}(x) y^{n}+q_{n-1}(x) y^{n-1}+\cdots+q_{0}(x) . u(\hat{x}, y)$ is for every fixed $\hat{x} \in(a, b)$ a polynomial in $y$ satisfying $|u(\widehat{x}, y)|<1$ in an interval of length $\geqslant 4$. This is possible only if the coefficient of $y^{n}<1$. For, if $T(y)$ is a polynomial whose leading coefficient is 1 and which deviates least from zero in $(c, d)$, we find $|T|_{\max }=$ $=2\left(\frac{k}{4}\right)^{n}$ (the Tschebycheff-polynomial). It follows that $|T|_{\max }>1$ if $k \geqslant 4$. Hence the leading coefficient of $u(\hat{x}, y)$ is absolutely less than one. Thus $q_{n}(x)$ has the property $\left|q_{n}(x)\right|<1$ for all $\hat{x} \in(a, b)$, which gives $q_{n}(\bar{x})=0$ if $\bar{x} \in N_{x}(a, b)$. Repeating this argument with an $x \in N_{x}(a, b)$ we find $\left|q_{n-1}(x)\right|<1$ in a set $P_{n-1} \subset(a, b)$. If we apply Fekete's theorem to the set $P_{n-1}$, which obviously contains $N_{x}(a, b)$, we get $N\left(P_{n-1}\right)=N_{x}(a, b)$ and we must have $q_{n-1}(\bar{x})=0$ if $\bar{x} \in N_{x}(a, b)$. Proceeding this way we finally get $u(\bar{x}, y)=0$ if $\bar{x} \in N_{x}(a, b)$. Hence theorem 6 is proved.

Theorem 7: $A$ set $C$ which contains an axis-parallel square, whose side $\geqslant 4$, cannot belong to $M$ or $M^{\prime}$.

Proof: We suppose that there exists an $I$-polynomial $u(x, y) \neq 0$ with $|u(x, y)|<1$ in $C$ and will show that we come to a contradiction. Put $u(x, y)=q_{n}(x) y^{n}+$ $+q_{n-1}(x) y^{n-1}+\cdots+q_{0}(x)$. In order that, for any fixed $x,|u(x, y)|<1$ will hold for every $y$ in an interval of length $\geqslant 4$, we must have $\left|q_{n}(x)\right|<1$ in $C$ according to the same reasoning as in theorem 6. But $\left|q_{n}(x)\right|<1$ is impossible in an interval of length $\geqslant 4$ and theorem 7 is proved.

Theorem 5-7 can be generalized in many ways. The number of variables can be increased. We can transform the sets by substitutions with integral coefficients in obviuos ways. The elements of the product-sets need not be an interval. Finally in theorem 5 and 6 we can deal with sets included in rectangles.

\section{REFEREN CES}

1. Loreniz, Bernstein Polynomials. Theorem 1.3.1, page 9.

2. Polya-Szegö, Aufgaben und Lehrsätze I. Aufgabe 11.

3. Ferete, M., Approximations par polynomes avec conditions diophantiennes. C. R. Acad. Sci. Paris 239. 1337-1339 and 1455-1457 (1954). 